\title{
EDMUS, a European database for multiple sclerosis
}

\author{
C Confavreux, D A S Compston, O R Hommes, W I McDonald, A J Thompson
}

\begin{abstract}
EDMUS is a minimal descriptive record developed for research purposes to document clinical and laboratory data in patients with multiple sclerosis (MS). It has been designed by a committee of the European Concerted Action for MS, organised under the auspices of the Commission of the European Communities. The software is user-friendly and fast, with a minimal set of obligatory data. Priority has been given to analytical data and the system is capable of automatically generating data, such as diagnosis classification, using appropriate algorithms. This procedure saves time, ensures a uniform approach to individual cases and allows automatic updating of the classification whenever additional information becomes available. It is also compatible with future developments and requirements since new algorithms can be entered in the programme when necessary. This system is flexible and may be adapted to the users needs. It is run on Apple and IBM-PC personal microcomputers. Great care has been taken to preserve confidentiality of the data. It is anticipated that this "common" language will enable the collection of appropriate cases for specific purposes, including population-based studies of MS and will be particularly useful in projects where the collaboration of several centres is needed to recruit a critical number of patients.
\end{abstract}

(F Neurol Neurosurg Psychiatry 1992;55:671-676)

There is a need to standardise morbidity records in patients with multiple sclerosis (MS). The use of a common language is an essential prerequisite in the establishment of a database which will provide descriptive details of the disease and allow rapid identification of appropriate patients for cross-sectional studies or therapeutic trials. The establishment of a database is one of the main themes of the European Concerted Action for Basic Research and Treatment in Multiple Sclerosis organised under the auspices of the Commission of the European Communities (CEC).

The Lyon MS database system, which has been developing since 1976, served as a basis. ${ }^{1}$ A workshop, Towards a European Database System for Multiple Sclerosis, was held in Lyon, France, 21-22 September 1990 to complete the design of standardised forms. An EDMUS Steering Committee, composed of members from the various countries of the European Economic Community (EEC), was also nominated. In 1991 the software was refined and tested by the EDMUS Steering Committee members. We present the conceptual and technical design of the EDMUS system, with special emphasis on the Basic Version, which could be considered a minimal descriptive record for any patient with MS. This version is now available and it is anticipated that it will be used in European collaborative studies in MS.

The EDMUS system: basic concepts

The aim of this database system is not to replace the clinical notes, as is the case for the North American COSTAR system, but to provide a minimal descriptive record for research purposes.

It is user-friendly and fast to facilitate regular and long term use, even in departments with limited facilities. In this respect, a minimal set of obligatory data has been defined. This does not exceed 15 items per patient and takes only a few minutes to complete. Additional complementary data may be entered for a more thorough description.

The description of the clinical manifestations of MS often relies on retrospective data provided by the patient, his relatives or general practitioner. This is usually applicable to the onset of the disease but may also be relevant to the earlier years of the disease. Consequently, in this system, priority has been given to symptoms rather than signs, to include both retrospective and prospective data.

One of the main advantages of computerised data processing is the ability to incorporate algorithms in the programme which allow automatic generation of data. Thus priority has been given to analytical data from which interpretations such as classification of the course, severity and diagnostic confidence of the disease, are computer generated. This procedure has many advantages. It is time saving for the clinician and gives an overview which may be used as a disease summary for any re-examination of the patient. It ensures a standardised way of classifying cases and provides an up-to-date classification of the case. Lastly, it can be made compatible with any future classification, as the data can be analysed using new algorithms.

Flexibility is another essential characteristic of the EDMUS system and both basic and extended versions are available according to the user's needs.

The programme has been developed on the 
standard database software OMNIS V (Blyth Software). It runs on both Apple and IBM-PC personal microcomputers and does not require sophisticated hardware. Apple computers of II LC, II Si, II Ci, PowerBook 140 and PowerBook 170 type are appropriate with a core memory size of 4 Megabytes, a hard disk of 40 Megabytes and either a monochrome or a high definition colour screen. IBM-PC and compatible computers must be based on INTEL 80386 or INTEL $80386 \mathrm{SX}$, with a $\geq 20 \mathrm{Mhz}$ clock speed, a similar core memory and hard disk as for Apple models. Apart from the standard operating system (MS-DOS level 3.xx), MS-WINDOWS 3.0x must be used.

Data which are entered include the names of the patients. This is necessary for any department using the system for its own patients as well as for multicentre studies since several records may have been registered from the same patient. Consequently, several safety measures have been taken to preserve confidentiality of the data, in accordance with the recommendations of the French Commission Nationale Informatique et Libertés (CNIL). Access to the EDMUS software and the files requires specific passwords. These may be updated at the convenience of the local database manager, that is, the person who is responsible for local use of the system. There are several levels for these passwords. The database manager level provides access to the various possible operations on the files and to named data. By contrast, the lowest level allows access to anonymous data only, and does not allow any modification to be done. An automatic procedure is available for suppressing the names of patients in the records when files are to be duplicated. Any centre must store duplicated named files for safekeeping. It is recommended that such copies might be limited in number to what is strictly necessary for this purpose and stored in an appropriate protected place. Physical protection is also required for the computer in which the named data are stored. Finally, any data exchange between centres or researchers must be made through anonymous files.

\section{The EDMUS form}

\section{General structure}

The form is made up of 2 sections. Section I is devoted to: 1) the patient's identification; 2) the MS background, with genetic data and the patient's medical history exclusive of MS; 3 ) the key-dates of the MS course in terms of relapses and onset of progression (fig 1); 4) the key-dates of the non-reversible disability; 5) general comments, with mention of available patient's biological samples, if any, and keywords which may be specifically coined for the patient and retrieved through the selection procedure of the programme (see below). It has a fixed length, that is, with newly available

Figure 1 The "MS Course Key-Dates" window.

This is devoted to the successive description of relapses and onset of progression, that is, the pivotal events which produce modifications in the symptoms (not signs only) of the disease. It is a part of Section I of the EDMUS form.

Type:

During the "no-sequelae period" of the relapsing-remitting phase, a distinction is made between: 1) the relapse at the onset of this period (RO);2) subsequent relapses with a well identified date of onset and a complete remission (RR); 3) relapses with complete remission but uncertain chronology $(R U)$. The latter option may be necessary for retrospective data.

During the "sequelae period" of the relapsing-remitting phase, a distinction is made between: 1) the relapse at the onset of this period (SO);2) subsequent relapses with a well identified date of onset and followed by a non-worsening $(S N)$, or a worsening (SW), or a doubtful worsening (S?) of pre-existing sequelae; 3) relapses with uncertain chronology (SU).

During the chronic progressive phase, a distinction is made between: 1) onset without any initial relapse (POP) and onset with an initial relapse (POR), which are mutually exclusive; 2) subsequent relapses with a well identified chronology (PR); 3) subsequent relapses with uncertain chronology (PU). The possible worsening effect of the relapse during the chronic-progressive phase is not taken into account, as it is often too difficult to assess.

Symptoms:

They are distributed in 14 categories, each of them with an appropriate abbreviation: lower extremity dysfunction (LE), upper extremity dysfunction (UE), sensory symptoms (SS), sphincter disturbance (SP), sexual disturbance (SX), facial motor (FM), facial sensory (FS), oculomotor (OM), vestibular and/or cochlear (VE), bulbar (BB), optic neuritis (ON), psychiatric symptoms (PS), mental deterioration (MT) and other symptoms not covered by the preceding categories (OT). Whenever symptoms related to the event to be described are unknown, the code "UK" is ticked. 
information during follow up, the original document is completed or, whenever necessary, modified but no extra sheets are used. The minimal set of Obligatory Data is identified by heavy framing. Finally, a "Wait and See" item has been prepared. It is used whenever the diagnosis of MS is doubtful irrespective of assignment in the Poser classification. $^{2}$

Section II describes the Current Status (CS) of the patient, that is, the state of the patient at any given date in the disease, and includes: 1) the current disability scores; 2) current immunological treatments, if any; 3) current symptoms and signs; 4) the results of current investigations including electrophysiology, CSF analysis, MRI and other imaging techniques. One CS form should be completed for every date under which the state of the patient is to be described. The CS can be documented retrospectively, whenever it is considered of interest to record clinical or paraclinical data relating to a previous date. It may also be documented prospectively, whenever the patient is seen for follow up. Consequently, section II is made up of additive CS forms, corresponding to the number of assessments collected during the follow up of the patient. In this way it provides a longitudinal description of the disease.

\section{The MS course key-dates form}

This form is devoted to a successive description of the relapses and the onset of progression, that is, the pivotal events which produce modifications in the symptoms (not signs only) of the disease. As shown in the diagram (fig 1), two distinct phases are considered. During the relapsing-remitting phase, the relapses are separated by periods of clinical inactivity. A relapse is defined ${ }^{3}$ as the occurrence of new symptoms, the re-appearance of former ones, or the worsening of current symptoms of at least 24 hours duration. It has a subacute onset; the initial phase is followed by a plateau and, usually, by improvement which may be complete. Transient worsening of symptoms associated with a rise in temperature is not considered as a relapse. Relapses must be separated by at least one month, and symptoms occurring within a month are considered as part of the same episode. Within the relapsing-remitting phase, $a$ no sequelae period and $a$ sequelae period may be considered. During the latter, the sequelae must produce at least a minimum ambulation-related disability or a significant not ambulation-related disability, such as, grade 3 on the EIS-DSS adapted scale (see below). The chronicprogressive phase is defined ${ }^{3}$ as continuous worsening of symptoms and signs of grade 3 or more on the EIS-DSS adapted scale (see below) for a minimum of six months, whether supervened by relapses or not. According to this scheme, there is a logical sequence in the description of the course. With time, the no sequelae period may be followed in the same patient by sequelae within the relapsing-remitting phase and then by chronic-progressive disease.
Specific definitions and abbreviations have been adopted for the characterisation of the relapses and the onset of progression. They are displayed in fig 1. For each event, the date of onset and concurrent symptoms should be specified. Fourteen categories of symptoms have been considered. They are displayed with the corresponding abbreviations in fig 1 . In the case of retrospective data, symptoms observed during the relapse or the onset of progression may be unknown. It is then possible to tick the appropriate code. In this part of the EDMUS form, the symptoms are entered in a concise way. If a more thorough description is desired at the clinical (or paraclinical) level, a Current Status form may also be completed relating to the same assessment.

\section{The disability scales}

The Kurtzke DSS scale ${ }^{4}$ has served as the starting model since it is widely used in clinical practice and research. However, it has been adapted to be unambiguous, notably by not mixing functional systems and by being quick to assess, even retrospectively. It is mainly based on symptoms and walking abilities. This EDMUS impairment scale (EIS-DSS adapted; Appendix 1) provides good inter-rater agreement (unpublished observations). The other disability scales which have been selected are Kurtzke's EDSS and functional systems scales $^{56}$ and the ambulation index. ${ }^{7}$

The automated functions of the EDMUS software

Cross-checking of the data

The windows which are displayed on the screen are directly derived from the EDMUS chart and, as the data is being entered, the user is automatically guided by the programme. For instance, the unmarried name and the pregnancy boxes will appear on the screen only if a female patient is being registered.

Consistency is maintained within any given window. For example, in the Course KeyDates window, whenever a relapse or the onset of progression is described, its type and date of onset are compared with any preceding events. In the case of inconsistency, a warning message appears and an appropriate correction is requested. A similar control is ensured for the Disability Key-Dates window where dates for the various disability levels must be entered in a logical chronological order.

Overall consistency is also checked automatically. With the exception of the birth date, no date can be entered if it precedes the date of the first relapse in cases with a remittent onset or the date of the onset of progression in cases with a progressive onset which has been registered in the Course Key-Dates window. Similarly, in the Current Status window, it is impossible to enter an EIS-DSS adapted disability level which would be lower than the non-reversible disability level already recorded in the Disability Key-Dates window.

\section{Generation of data}

At the same time as analytical data are registered, further information is automatically 
generated and updated as additional information is entered. For instance, the date of the last examination is deduced from the latest date among the Course Key-Dates, the Disability Key-Dates and the Current Status windows. This allows an appropriate calculation of the duration of disease and length of follow up for any case. Similarly, there are algorithms for the classification of the disease course, severity and confidence of diagnosis.

The classification of the disease course is made through the Course Key-Dates window according to established criteria. ${ }^{8}$ The remittent forms show events (relapses) in the exacerbating-remitting phase and none (onset of progression and subsequent relapses, if any) in the chronic-progressive phase, while the remittentprogressive forms show events in both phases, and the progressive forms displays the chronicprogressive phase only. This latter category is subdivided into with and without superimposed relapses.

The classification of the severity of disease is generated from the Disability Key-Dates window according to a previous grade proposal. ${ }^{8}$ When severe non-reversible disability has developed (grade $\geq 7$ on the EIS-DSS adapted scale), the interval to severe disability is calculated from the date of onset of the disease, that is, the date of the first event in the Course KeyDates window. The following definitions are applied:

Hyperacute form: interval to severe non-reversible disability $<5$ years.

Acute form: interval to severe non-reversible disability $=5-10$ years.

Subacute form: interval to severe non-reversible disability $=10-15$ years.

Intermediate form: interval to severe non-reversible disability $\geq 15$ years.

Benign form: residual disability less than grade 3 with a disease duration greater than 20 years.
Not assessable form: case that fails to fulfil the preceding categories.

The generation of the diagnosis classification is more complex. It is displayed on the Diagnosis Synopsis window (fig 2) which has been designed to display items relevant to the generation of the diagnosis using the Poser criteria. ${ }^{2}$ These items are picked up from the whole case record. In this way, it is very easy to know if a given examination has already been done, what has been its diagnostic value, and the rationale for doing or repeating it. These items are grouped according to the 3 criteria of the Poser classification: 1) dissemination of lesions in time; 2) dissemination of lesions in space, evidence of which may be clinical or paraclinical (evoked potentials, CT scan or MRI examinations); 3) quantitative and/or qualitative abnormalities of immunoglobulins in the cerebrospinal fluid.

The criteria for dissemination in time and the date of completion are generated directly from the second event, if any, which has been entered in the Course Key-Dates window. For dissemination in space, several steps are followed. First, relevant data are extracted from sections I and II of the record. The involvement of the neuraxis is demonstrated clinically whenever neuraxis symptoms and/or signs have been entered in the Course Key-Dates, Disability Key-Dates or Current Status windows. It is also demonstrated electrophysiologically when brainstem auditory, somatosensory or motor evoked potentials provide positive results. The earliest date of positivity among these items serves as the date of completion for the neuraxis item. A similar procedure is followed for the optic nerve. The imaging item is generated as positive as soon as the MRI examination (or the CT scan for earlier patients) has been entered in a Current Status window as strongly suggestive of MS according to Paty's criteria. ${ }^{9}$ In a second step, the criteria

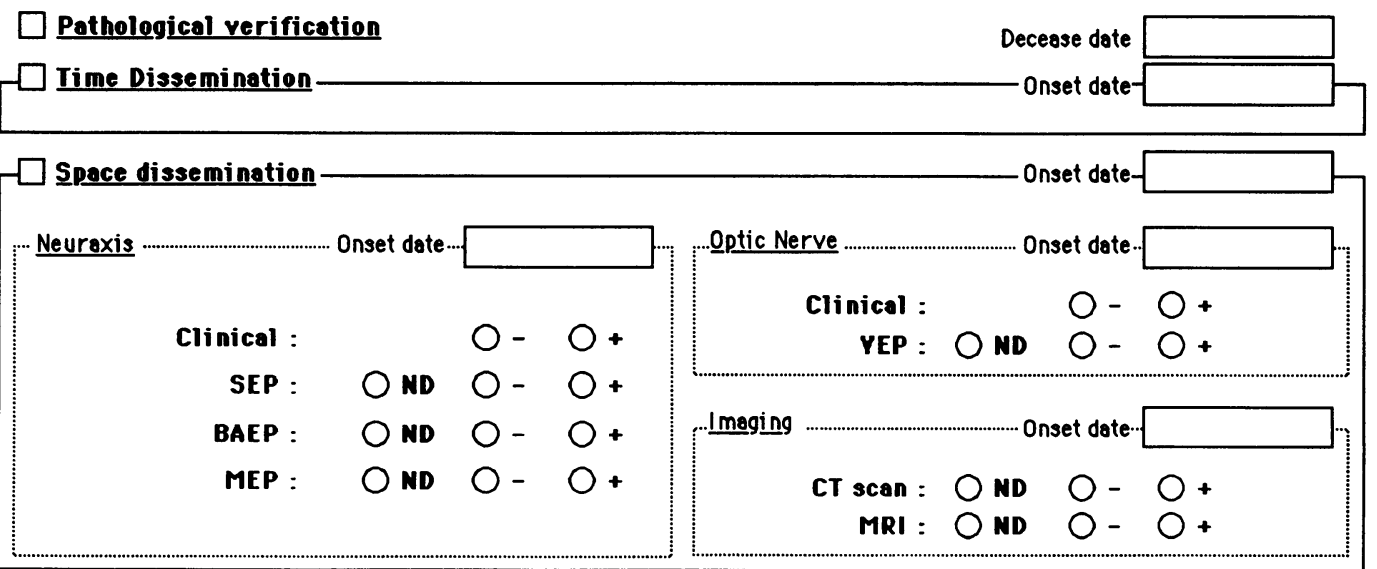

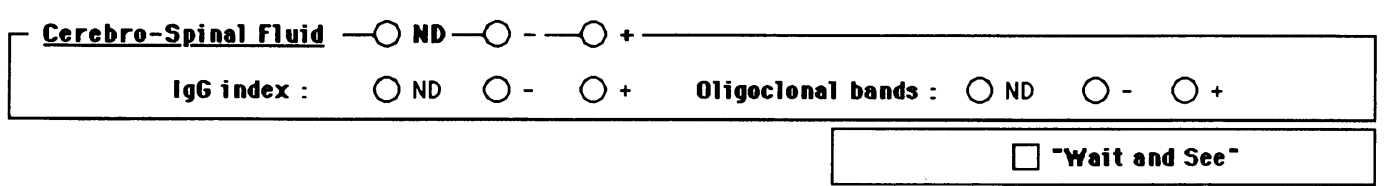

Figure 2 The "Diagnosis Synopsis" window. This is a window of the EDMUS programme but not a part of the EDMUS form. It displays data which are automatically extracted from the whole case record, such as, Sections I and II of the EDMUS form. From these data, the diagnosis is automatically classified in accordance with the Poser's criteria (see text). SEP = somato-sensory evoked potentials; $B A E P=$ brainstem auditory evoked potentials; $M E P=$ motor evoked potentials; VEP = visual evoked potentials; MRI = magnetic resonance imaging; ND $=$ not done. 
for dissemination in space and date at which this was established are completed whenever any of the following combinations from the neuraxis, optic nerve and imaging items is obtained: neuraxis + optic nerve + imaging; neuraxis + optic nerve; neuraxis + imaging; optic nerve + imaging. The $C S F$ criteria are generated as positive as soon as the presence of oligoclonal banding has been entered and/or the IgG index ${ }^{10}$ is higher than 0.70 in a Current Status window. The earliest date of CSF positivity serves as the date of completion of the CSF item.

Finally, the time, space and CSF criteria are automatically combined by the programme to generate and classify the diagnosis in accordance with Poser's guidelines. ${ }^{2}$

Clinically definite case: "time + space" whatever the "CSF" result.

Laboratory-supported definite case: "time + CSF" or "space + CSF".

Clinically probable case: "time" only or "space" only.

Laboratory-supported case: "CSF" only.

Suspected case: case that fails to fulfil the preceding categories.

Case selection and export facilities

A selection of the records from the whole file according to any criterion, isolated or in combination, is possible through the Selection window (fig 3 ). The list of the selected records may be printed. Data from these records may be transferred to standard softwares, for instance EXCEL (Microsoft Corporation) or SPSS (SPSS Incorporation) for statistical analysis.

\section{Present and future developments}

Diffusion of the EDMUS system

The first version of the EDMUS system is now available and may be ordered through the Clinique de Neurologie, Hôpital Neurologique, Lyon, France (cost $=600$ ECUs). Colleagues are advised to contact the EDMUS Steering Committee member in their own country for further information. The Lyon Clinique de Neurologie will act as EDMUS managing office and will provide assistance in several areas: knowledge of the system, local implementation of the system, conversion (if possible) of previously collected data to the EDMUS system, and long call assistance via Fax. It will also act as an EDMUS coordinating centre. Such a structure is necessary for several reasons: 1) to distribute, maintain and continue to develop the system; 2) to promote and coordinate collaborative studies; 3 ) to establish a connexion with tissue banking facilities. In this respect, it may be noted that, for any record on the EDMUS system, it is possible to register the availability of biological material, either DNA, cells, CSF, serum or brain tissue. Any local EDMUS user will have the possibility of forwarding anonymous files to
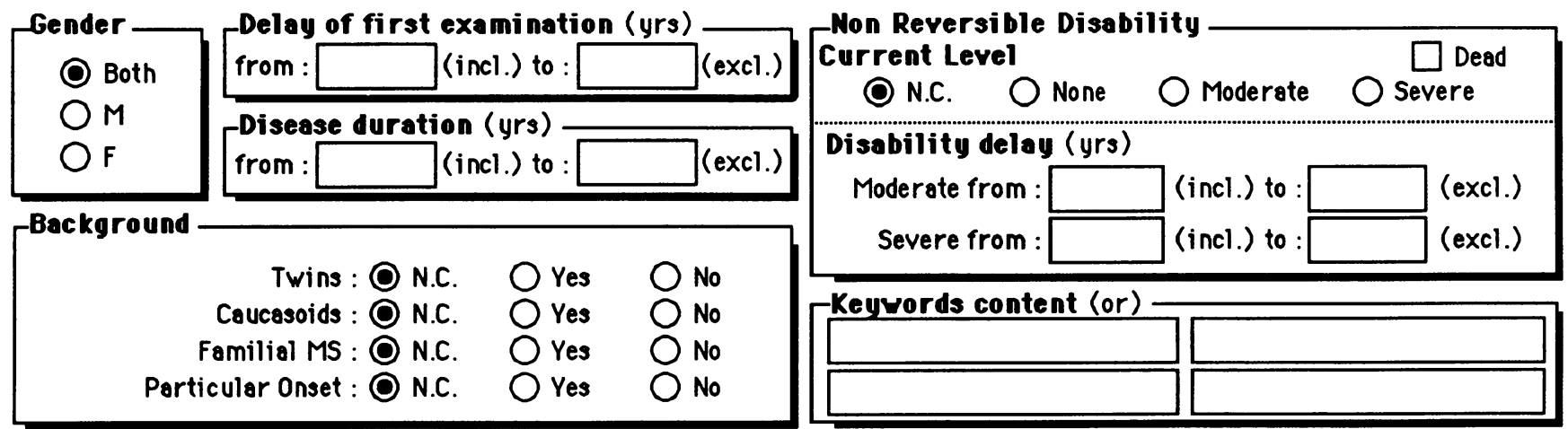

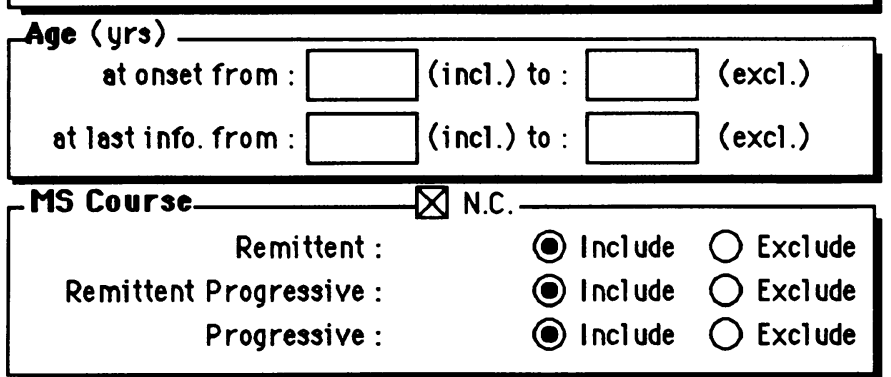

$\begin{aligned} & \text { Onset Symptoms } \\ & \text { Long Tracts: } \mathrm{O} \text { N.C. } \bigcirc \text { Include } \bigcirc \text { Exclude } \square \text { /solated } \\ & \text { Brainstem: } \bigcirc \text { N.C. } \bigcirc \text { Include } \bigcirc \text { Exclude } \square \text { /solated } \\ & \text { Optic Neuritis: } \bigcirc \text { N.C. } \bigcirc \text { Include } \bigcirc \text { Exclude } \square \text { /solated }\end{aligned}$

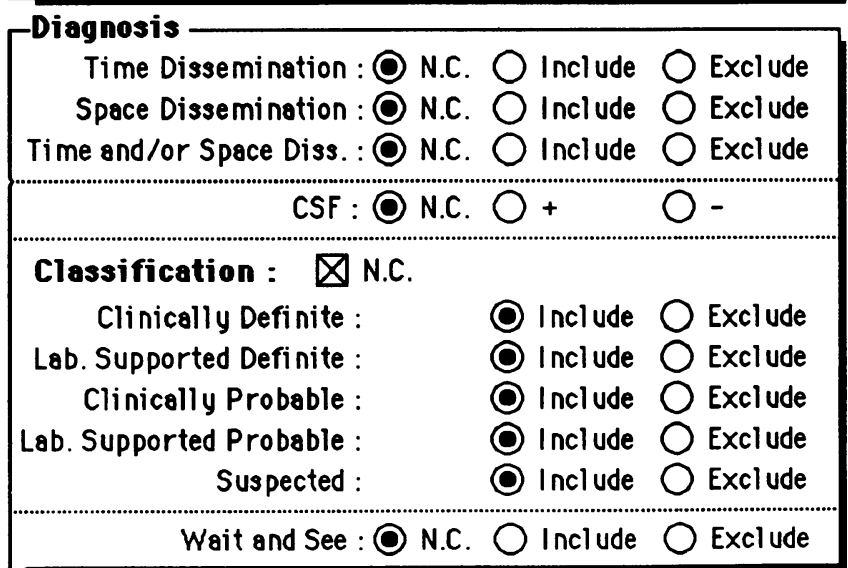

Figure 3 The "Selection" window is a window of the EDMUS programme but not a part of the EDMUS form. It displays the pre-established criteria which may be used, in isolation or in combination, for the selection of records from the whole file.

$M=$ male; $F=$ female; yrs $=$ years; incl $=$ inclusive; excl $=$ exclusive; $N C=$ not concerned. Delays and durations are calculated by comparing the date of onset of the disease and the date of onset of the relevant item. For instance, in "delay of first examination" calculations are made from the date of onset of the disease to the date of first examination of the patient in the department, data which are present in Section I of the EDMUS form. It is then possible to select records for example, with a minimum (inclusive) of 6 months and a maximum of 10 years (exclusive) for the "delay of first examination" by entering 0.5 and 10 in the appropriate boxes. "Key-words": by entering a given key-word in the appropriate box, it is possible to select records for which such a key-word has been entered in the "Comments" part of Section I of the EDMUS form. 
the coordinating centre via a disquette or modem. The coordinating centre will provide the users with the general characteristics of the files which have been collected and help in the retrieval of records when requested. Access to and disposal of any given record will be allowed only with the formal agreement of the EDMUS user in charge of this record. Confidentiality of the data will also, be preserved at this level. In these various tasks, the coordinating centre will continue to be assisted and permanently controlled by the EDMUS Steering Committee members.

\section{Development of future versions of the EDMUS system}

The EDMUS system will evolve in agreement with the users and the steering committee members recommendations. For example, new algorithms could be incorporated into the system, if considered relevant. Moreover, besides the present Basic Version of the EDMUS system, it is necessary to prepare several extended versions aimed at specific studies. Thus an MRI Extended Version is anticipated for MRI monitoring treatment of MS, in collaboration with the MRI Committee of the European Concerted Action for Multiple Sclerosis ${ }^{11}$; a Register Version, displaying only the minimal set of obligatory data of the Basic Version, is planned for epidemiological surveys, and a Genetic Version for GAMES (Genetic Analysis of Multiple Sclerosis in the European Society).

\section{Collaborative activities}

While the EDMUS system may be used within individual departments, the aim of this project is to share data from different centres and promote collaboration between them. The members of the EDMUS Steering Committee have already agreed to register systematically in the EDMUS system all newly diagnosed cases of MS in their departments, to test the reliability of the system. A collaborative prospective study of the relationship between MS and pregnancy is in preparation. There are many other areas to which the system may be adapted, including genetic analysis of twins and multiplex families, MS in immigrants to Europe, longitudinal MRI studies and therapeutic trials. Such collaborative European studies within the European Concerted Action for MS will be greatly enhanced through the use of this commonly agreed language. It is hoped that the National MS Societies, the European MS Platform and the European Economic Community will contribute to setting up and maintaining a central facility which could be pivotal in all future research in MS throughout Europe.

The continuous contribution of Mr Albert Biron, Département Informatique des Hospices Civils de Lyon, in the design and the Informatique des Hospices Civils de Lyon, in the design and the Azerad, Option Performance, in the development of the Azerad, Option Performance, in the development of the
programme have been appreciated. The Committee gratefully programme have been appreciated. The Committee gratefully acknowledge the support of the European Charcot Foundation, Hospices Civils de Lyon, Association pour la Recherche sur la
Sclérose En Plaques (ARSEP), Ligue Française contre la Sclérose En Plaques (ARSEP)
Sclérose En Plaques (LFSEP).
1 Confavreux C, Moreau T, Wolfson C, et al. Natural history and prognosis in multiple sclerosis: the Lyon MS data-
base. In: Battaglia MA, ed. Multiple sclerosis research. Amsterdam: Elsevier 1989:199-203.

2 Poser CM, Paty DW, Scheinberg L, et al. New diagnostic criteria for multiple sclerosis: guidelines for research criteria for multiple sclerosis: guidelines

3 Schumacher GA, Beebe G, Kibler RF, et al. Problems of experimental trials of therapy in MS: report by the panel on the evaluation of experimental trials of therapy in MS. Ann NY Acad Sci 1965;122:552-68.

4 Kurtzke JF. On the evaluation of disability in multiple sclerosis. Neurology 1961;11:686-94.

5 Kurtzke JF. Further notes on disability evaluation in multiple sclerosis, with scale modifications. Neurology 1965;15:654-61.

6 Kurtzke JF. Rating neurological impairment in multiple sclerosis: an expanded disability status scale (EDSS). Neurology 1983;33:1444-52.

7 Hauser SL, Dawson DM, Lehrich JR, et al. Intensive immunosuppression in progressive multiple sclerosis: a randomized, three-arm study of high-dose intravenous cyclophosphamide, plasma exchange, and ACTH. N Engl cyclophosphamide, plasm

8 Confavreux C, Aimard G, Devic M. Course and prognosis of Multiple Sclerosis assessed by the computerized data processing of 349 patients. Brain 1980;103:281-300.

9 Lee KH, Hashimoto SA, Hooge JP, et al. Magnetic resonance imaging of the head in the diagnosis of multiple sclerosis: a prospective 2 year follow up with comparison of clinical evaluation, evoked potentials, oligoclonal banding, and CT. Neurology 1991;41:657-60.

10 Delpech B, Lichtblau F. Etude quantitative des immunoglobulines $G$ et de l'albumine du liquide céphalorachidien. Clin Chim Acta 1972;37:15-23.

11 Miller DH, Barkoff F, Berry I, Kappos L, Scotti G, Thompson AJ. Magnetic resonance imaging in monitoring the treatment of multiple sclerosis: Concerted Action
Guidelines. F Neurol Neurosurg Psychiat 1991;54:683-8.

\section{APPENDIX}

Appendix 1: The EDMUS Impairment Scale (DSS adapted):

EIS (DSS adapted)

Score

0 Normal findings on neurological examination.

1 No disability. Minimal signs on neurological examination.

2 Minimal and not ambulation-related disability. Able to run.

3 Unlimited walking distance (WD) without rest, but unable to run; or a significant not ambulationrelated disability.

4 Walks without aid. Limited WD, but $>500$ meters without rest.

5 Walks without aid. WD $<500$ meters without rest.

6 Walks with uni or bilateral support. WD $<100$ meters without rest.

7 Home restricted. A few steps with wall or furniture assistance. WD $<10$ meters without rest.

8 Chair restricted. Unable to take a step. Some effective use of arms.

9 Bedridden and totally helpless.

10 Death due to MS.

Appendix 2: List of the EDMUS Steering Committee members

Belgium and Luxemburg: Dr Richard Gonsette, Melsbroek.

Denmark: Dr Niels Koch-Henriksen, Odense.

Germany: Professor Hans-Peter Hartung, Würzburg.

France: Professor Christian Confavreux (President), Lyon; Dr Annick Alperovitch, Villejuif; Professor Gilles Edan, Rennes.

Ireland: Dr Michael Hutchinson, Dublin.

Italy: Professor Giancarlo Comi, Milan.

The Netherlands: Professor Otto Hommes (Projectleader of the European Concerted Action for MS), Nijmegen.

Portugal: Professor Antonio Magalhaes, Lisboa.

Spain: Dr Oscar Fernandez, Malaga.

Switzerland: Dr Ludwig Kappos, Basel.

United Kingdom: Professor Alastair Compston, Cambridge; Dr Alan Thompson, London. 